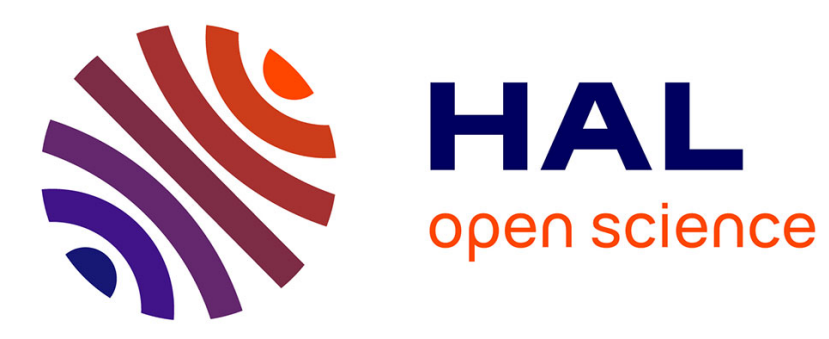

\title{
Effects of Hand Feedback Fidelity on Near Space Pointing Performance and User Acceptance
}

\author{
Andreas Pusch, Olivier A.J. Martin, Sabine Coquillart
}

\section{To cite this version:}

Andreas Pusch, Olivier A.J. Martin, Sabine Coquillart. Effects of Hand Feedback Fidelity on Near Space Pointing Performance and User Acceptance. ISVRI 2011 - International Symposium on Virtual Reality Innovation, Mar 2011, Singapore, Malaysia. pp.97-102, 10.1109/ISVRI.2011.5759609 . hal00642174

\section{HAL Id: hal-00642174 \\ https://hal.science/hal-00642174}

Submitted on 9 Jan 2013

HAL is a multi-disciplinary open access archive for the deposit and dissemination of scientific research documents, whether they are published or not. The documents may come from teaching and research institutions in France or abroad, or from public or private research centers.
L'archive ouverte pluridisciplinaire HAL, est destinée au dépôt et à la diffusion de documents scientifiques de niveau recherche, publiés ou non, émanant des établissements d'enseignement et de recherche français ou étrangers, des laboratoires publics ou privés. 


\section{Effects of Hand Feedback Fidelity on Near Space Pointing Performance and User Acceptance}

\author{
Andreas Pusch ${ }^{1,4}$ \\ INRIA Grenoble Rhône-Alpes - LIG \\ 655, av. de l'Europe \\ 38334 St. Ismier Cedex, France
}

\author{
Olivier Martin ${ }^{2,5}$ \\ INRIA Grenoble Rhône-Alpes - LIG \\ 655, av. de l'Europe \\ 38334 St. Ismier Cedex, France
}

\author{
Sabine Coquillart ${ }^{3}$ \\ INRIA Grenoble Rhône-Alpes - LIG \\ 655, av. de l'Europe \\ 38334 St. Ismier Cedex, France
}

\begin{abstract}
In this paper, we report on an experiment conducted to test the effects of different hand representations on near space pointing performance and user preference. Subjects were presented with varying levels of hand realism, including real hand video, a high and a low level 3D hand model and an ordinary 3D pointer arrow. Behavioural data revealed that an abstract hand substitute like a $3 \mathrm{D}$ pointer arrow leads to significantly larger position estimation errors in terms of lateral target overshooting when touching virtual surfaces with only visual hand movement constraints. Further, questionnaire results show that a higher fidelity hand is preferred over lower fidelity representations for different aspects of the task. But we cannot conclude that realtime video feedback of the own hand is better rated than a high level static 3D hand model. Overall, these results, which largely confirm previous research, suggest that, although a higher fidelity feedback of the hand is desirable from an user acceptance point of view, motor performance seems not to be affected by varying degrees of limb realism - as long as a hand-like shape is provided.
\end{abstract}

Index terms: H.1.2 [Models and Principles]: User/Machine Systems---Human Factors; H.5.1 [Information Interfaces and Presentation]: Multimedia Information Systems---Artificial, Augmented, and Virtual Realities; I.3.6 [Computer Graphics]: Methodology and Techniques---Interaction Techniques

Additional keywords: Near space interaction, co-location, limb attribution, perception, video see-through head-mounted display, hand displacement, visuo-proprioceptive sensory conflict

\footnotetext{
andreas.pusch@imag.fr

olivier.martin@ujf-grenoble.fr

sabine.coquillart@inria.fr

4 Currently working at: IIHM - UJF - LIG, 110, av. de la Chimie - BP 53, 38041 Grenoble Cedex 9, France

5 Currently working at: GIPSA-lab - UJF, 961, rue de la Houille Blanche BP 46, 38402 Grenoble Cedex, France
}

\section{INTRODUCTION}

Object selection is a frequent task in human-computer interaction. In particular, when interacting with 3D user interfaces, vision of the own hand or its representation plays a decisive key role in the context of an intuitive direct control. The "feeling of ownership of a limb" [1, 2] helps to understand perceived actions as selfgenerated, since they typically match sensory feedback patterns predicted by the internal model of action [3]. The hand representation may appear faithful / reliable, if it exhibits biologically plausible motions $[4,5]$ and a natural / familiar look $[6,7]$. A higher fidelity visual hand feedback has further been shown to lead to a stronger visuo-proprioceptive integration [8] and to reveal shorter reaction and movement times compared to a lower fidelity hand feedback [9]. In a recent study, the effectiveness of the rubber hand illusion [10], when invoked in Virtual Reality (VR), depended also on visual limb faithfulness [11]. Another important prerequisite for a perceptually convincing interaction within hand's reach is co-location. This notion refers to the spatial visual and proprioceptive alignment of an interacting limb. A qualitatively and quantitatively beneficial direct control $[12,13]$ is conveyed as well as a stronger sense of presence [14]. The resulting self attribution [10] supports the natural continuous observation and correction loop responsible for compensating for pointing errors and / or target modifications [15], also in VR settings [16].

We have set up an experiment on top of a system similar to the one used in [17]. It is essentially based on a tracked (i.e., head and hand optically tracked at 6 degrees of freedom) Augmented Virtuality (AV) environment using a video see-through (VST, 640 x 480 video resolution) head-mounted display (HMD, 800 x 600 display resolution). This configuration permits to control the 3D location of the live stereo video feedback of the hand embedded into an otherwise virtual scene by displacing the carrier objects textured with the captured image data. In our study, we will focus on subjective user preference and objective behavioural effects of different levels of hand representations during pointing-like near space interaction / selection.

\section{Hypotheses}

It has been stated in different studies that subjects often preferred hand representations at higher visual fidelity levels. This is an important fact from an user-centred design / interaction ergonomics viewpoint. But the degree of realism has rarely been assessed, in particular regarding the context of this paper.

Hypothesis 1 focusses on the objective influence of the level of hand representations on behaviour in a goal-directed pointing task. With respect to related studies, we expect that the higher the fidelity of the hand feedback the more robust is limb attribution / perceptual reliance (i.e., perceptual link to the corresponding limb). Motor performance should thus improve compared lower fidelity representations. More precisely, first, better final pointing stability is assumed, if the realism level of the hand feedback increases (i.e., towards the own hand). Stability is considered an accuracy measure indicating the stimuli integration performance 
of the sensorimotor system. Second, we think that an intuitive feeling of control can further be reflected by the extent of virtual object penetration with the real hand while the visual hand rests on the object's surface. That is, higher feedback fidelity may lead to less target overshooting until the detection of the touching event.

Hypothesis 2 addresses effects on the subjective experience during virtual object touching within hand's reach. In agreement with previous research on VR limb realism and sense of presence, we expect that a high level representation such as stereo video feedback of the own hand will be preferred over other classical hand representations or avatars (i.e., detailed 3D hand model, simplified 3D hand model and ordinary 3D pointer arrow, see Fig. 1). This advantage should hold for different aspects of a virtual surface touching scenario, including hand visualisation quality, final pointing accuracy, hand movement naturalness and overall comfort. User acceptance may decrease with lower hand realism levels.
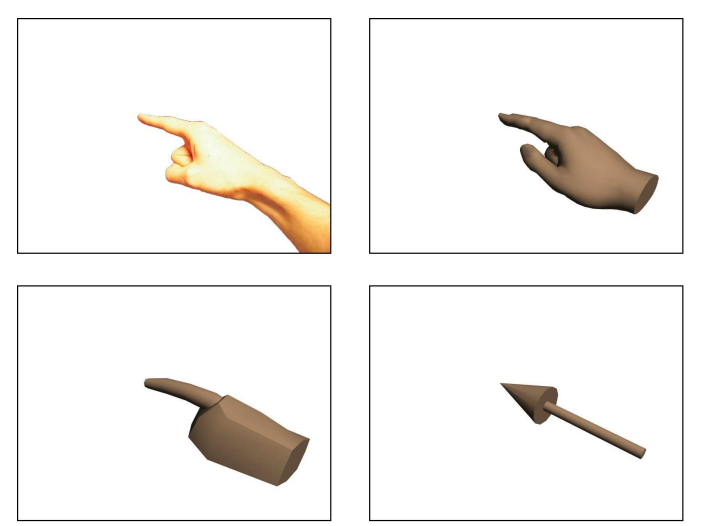

Figure 1: Hand representations used during the experiment (i.e., upper left: Video feedback, upper right: Detailed 3D model, lower left: Simplified 3D model, lower right: 3D pointer model).

Reaching duration and hand trajectory length will be observed as well between the moments where the hand enters the field of view and actually reaches the target. These measures may be indicators for action optimisation and economics. However, the effective hand viewing space of the VST HMD used in the experiment is rather small (i.e., about 43 degrees horiz. and 27 degrees vert.). Considering the relatively short visible hand transport phase from the rest position to the targets, effects may be marginal, but yet insightful.

\section{Subjects}

Sixteen adult volunteers (i.e., 20 - 40 years old, 7 female, 9 male) participated in the study. None of them reported serious vision problems (i.e., either normal or corrected to normal vision). A few subjects had some prior non-expert knowledge on Augmented Reality (AR), AV, VR and / or human perception. All were naive about the study's purpose and had never used the experimental setup.

\section{Methods}

In this section, we will present the experiment's factorial design, the pointing task to be performed, the administered questionnaire, as well as all relevant details on the data acquisition and analysis.

\subsection{Factorial design}

The experiment followed a 4 × 2 factorial design of which factor one specifies the number of hand representations (see Fig. 1) and factor two the number of pointing target locations on a cube surface (see Fig. 2).

All 3D hand models, including the arrow, had a common visual appearance in terms of size and a uniform skin-like shading. Additionally, they were displayed at six degrees of freedom according to the real hand tracking. The given video-tovirtual-world lag of about $50 \mathrm{~ms}$ was simulated for the purely virtual scenes.

To prevent fast hand movement adaptation, we decided to use more than one target location. Two sufficiently separated targets (i.e., top near and bottom far, $3 \mathrm{~cm}$ edge length, see Fig. 2, right) were expected to meet this requirement. However, advanced behavioural analyses may also be possible when varying the target characteristics in this manner.
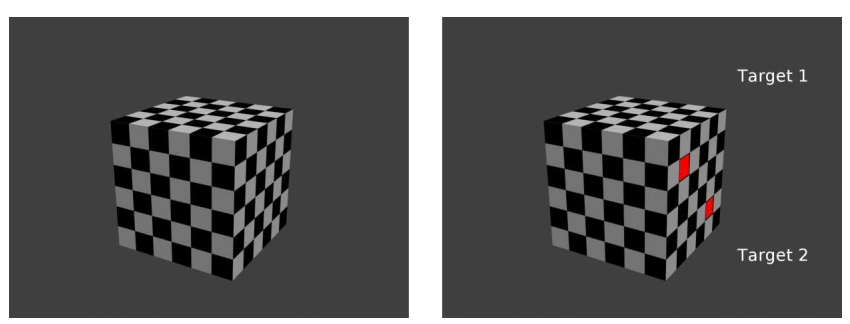

Figure 2: Virtual scene (left, cube: $20 \mathrm{~cm}$ edge length), with target locations (right).

In sum, there were eight conditions which had to be randomly distributed and equally weighted over the duration of the experiment to avoid any effect carry over. An $8 \times 8$ random latin square was generated to assure balanced trial sets. Each participant had to perform 16 repetition per condition making up 128 trials in total.

\subsection{Pointing task}

To be able to present the cube and thus the pointing targets at a similar relative height, the subject's shoulder was considered as the reference. The VST HMD was adjusted to the eye distance by shifting the eyepieces accordingly. A clear view of both display images had to be confirmed before continuing. Further, the rest position between trials (see Fig. 3, left) and the grasping pose for pointing and holding the hand tracking device (see Fig. 3, right) were explained. The sensitive point for interaction was calibrated to the top of the index finger.
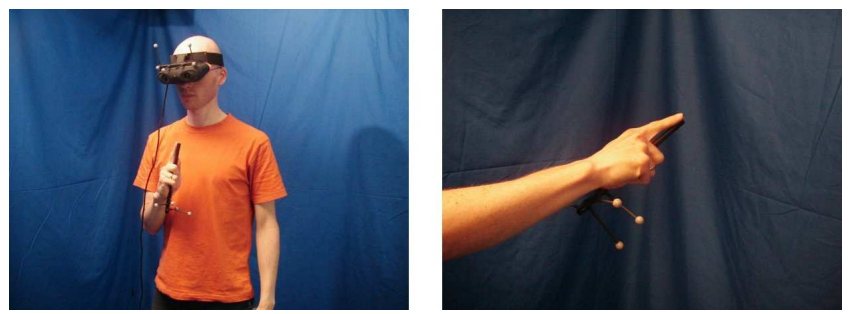

Figure 3: Rest position (left), grasp and pointing pose (right).

Regarding the actual pointing task, subjects were asked to line up on a defined position looking towards the blue-covered walls of the room. They were instructed to use the right index finger in order to touch the centre of the red square that would appear on the right side of the cube (see Fig. 4).

At the moment the target appears, a simultaneous acoustic trigger notification was played back (i.e., first beep). Subjects were told to have four seconds to do the pointing and to perform a precise rather than a rapid movement. Once they thought they had touched the target's centre, subjects had to return to the rest position. No other contact cues were presented. An acoustic trial end notification (i.e., second beep) would be heard at the same 
time the target disappears. This procedure recurred 128 times per subject, with a relaxation break of 5 minutes at the first half.

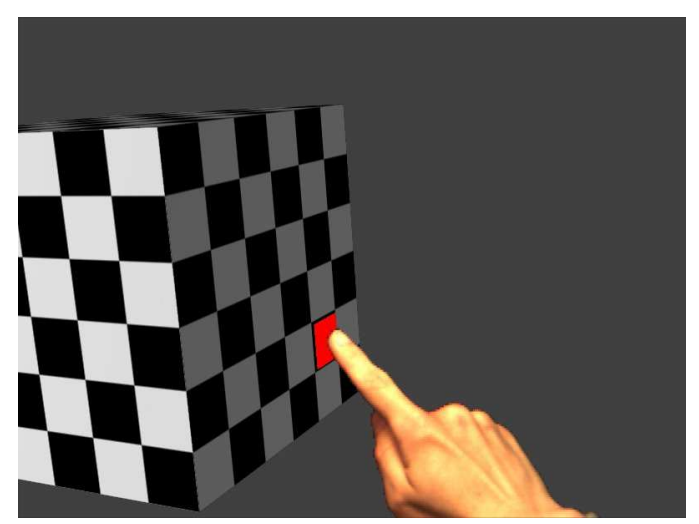

Figure 4: Application screenshot of a user touching the target (i.e., VST HMD view).

The hand feedback was visually constrained to the object surface using the rubber band method (RB) [18] both during contacts and on continued entering. This method minimises the visual offset between the real and the visual hand position. On release from the surface, that is, if the subject moves his hand away from the cube, we applied the incremental motion method (IM) [18] which maximises motion coherence and thus conveys a natural feeling of control (see also Fig. 5). The system underneath has been adopted from [17].

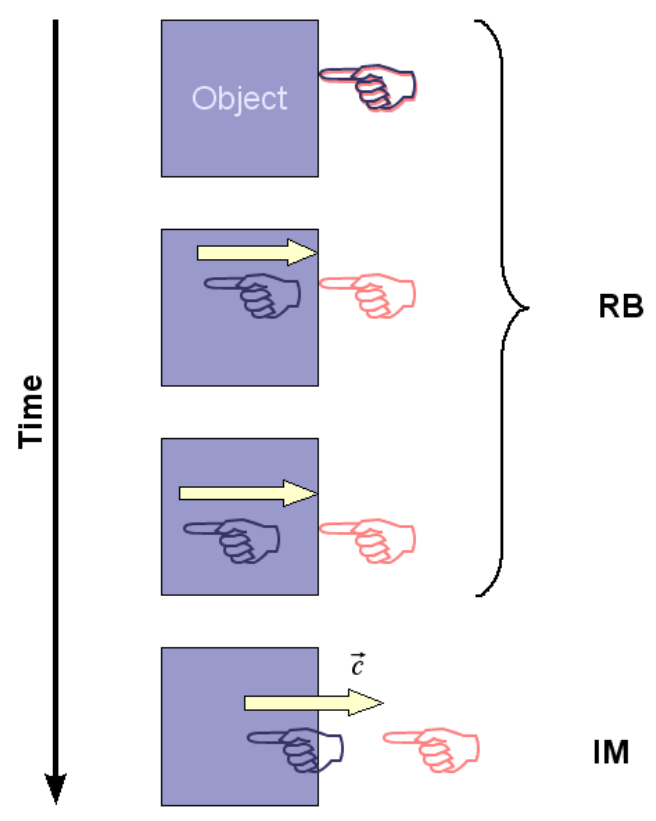

Figure 5: RB-IM process on surface entrering and release (dark blue: Real hand, salmon: Visual hand).

\subsection{Questionnaire}

A sequential questionnaire (see below) was given to the participants immediately after the pointing task was done. It contained consecutive open questions as well as a subjective hand representation evaluation section. It was not allowed to return to previously completed pages. Subjects were free to give written comments to any question. Discussions were only accepted in cases of comprehension problems.

Part one of the questionnaire focused on the differences perceived between trials over the whole experiment. If differing conditions were remembered, their total number, the globally preferred, supporting or even interfering ones had to be indicated and the sensation be explained. The next set of questions asked more precisely for the recognised hand representations, including their number and the subjective preference. A sketchy drawing of each recalled hand type was requested.

The second part mainly consisted of a multi-level hand representation evaluation. Subjects were shown images of the four hand representations used during the experiment. They had to assess the visualisation quality, the final pointing accuracy on the target, the naturalness of the hand movement or transport towards the red squares and the overall comfort while performing the tasks. Each aspect had to be evaluated on a 5-point scale (i.e., 1: Best to 5: Worst), as a function of the visual hand representation. A justification was required for the overall comfort assessment. General remarks could be given in the end of the questionnaire.

The items of the second part in detail:

1. Visual hand representations used for pointing (1: Very good => 5: Bad).

2. Final pointing accuracy on the target as a function of the visual hand representation (1: Very good $=>5: \mathrm{Bad}$ ). $<$ Page break>

3. Naturalness of the hand movement / transport towards the target as a function of the visual hand representation (1: Very good $/$ intuitive $=>5:$ Bad $/$ abstract).

4. Overall comfort while performing the tasks as a function of the visual hand representation (1: Comfortable $=>$ 5: Uncomfortable).

5. Explanation of the reasons for the best AND the worst assessment.

6. General remarks.

\subsection{Data acquisition and analysis}

For the behavioural analysis, we used head and hand tracking information recorded at approximately $60 \mathrm{~Hz}$. A first processing of this raw data yielded specific action events (i.e., entering the field of view, approaching the cube surface, stabilising the finger on the target and releasing the visual hand from the cube surface). The resulting dependent variables used for statistics were:

1. Coarse hand oscillation around the target (i.e., repeated visual contacts before returning to the rest position).

2. Target entering depth (i.e., maximum penetration perpendicular to the red square before returning to the rest position).

3. Hand movement duration (i.e., time between entering the field of view with the visual hand and stabilising it on the final target).

4. Hand trajectory length (i.e., path length between entering the field of view with the visual hand and stabilising it on the final target).

From the questionnaire, the hand representation assessment part was considered for the analysis. Other responses and comments served only as source for a clearer interpretation of the other results.

Behavioural and subjective evaluation data was analysed using descriptive statistics (i.e., mean and standard deviation, $\mathrm{SD}$ ), followed by a repeated measures Analysis of Variance (ANOVA) and, if adequate, correlation and / or post-hoc tests (e.g., Pearson's product moment correlation and / or pairwise comparison or Fisher's Least Significant Difference, LSD, resp.). 


\section{Results}

Because of continual reaching errors in 4 subjects, they were excluded from the analysis. Apart from that, a few trials had to be removed, mostly for technical reasons (e.g., corrupted tracking data, malfunctions of the system or the simulation).

\subsection{Coarse hand oscillation around the target}

When a subject touched a target for the first time, a counter was launched. All subsequent touching repetitions led to counter increments as long as the current trial's data acquisition ran. It is hence a measure for final pointing stability and so for terminal hand movement guidance quality.

There was no effect of the hand representation on the coarse pointing accuracy found $(\mathrm{F}(3,33)=0.8 ; \mathrm{p}>0.5)$. The target location effect was also not significant $(F(1,11)=3.47 ; p>0.09)$, although touching stability appeared to be more than 7 times higher on the far target (i.e., $\mathrm{SD}_{\text {far }}=0.009$ vs. $\mathrm{SD}_{\text {near }}=0.065$ ).

\subsection{Target entering depth}

This variable reflects the maximum target penetration before subjects decided to move their hand back to the rest position. Thus, the target entering depth tells us something about the lateral hand position estimation error or touching overshooting. The visual hand was always constrained to the cube's surface.

Analysis yielded a significant effect for both the hand feedback $(\mathrm{F}(3,33)=2.89 ; \mathrm{p}<0.05)$ and the target location $(\mathrm{F}(1,11)=15.73 ; \mathrm{p}<0.003)$. Regarding the hand representation, a post-hoc LSD test (i.e., pairwise comparison) revealed a significantly smaller target entering depth of the real hand video $(\mathrm{p}<0.021)$ and of the simplified 3D hand model $(\mathrm{p}<0.015)$ each compared to the ordinary $3 \mathrm{D}$ pointer arrow (see Fig. 6). The detailed 3D hand model was situated at an intermediate level without any statistically relevant performance variation. Correlation effects were not found, neither for the target factor in general $\left(\mathrm{r}^{2}=0.005 ; \mathrm{t}=0.72 ; \mathrm{p}>0.4\right)$ nor for any specific target $\left(r^{2}{ }_{\text {near }}=0.008 ; \mathrm{t}=0.59 ; \mathrm{p}>0.5\right.$ and $\left.\mathrm{r}_{\text {far }}=0.004 ; \mathrm{t}=0.44 ; \mathrm{p}>0.6\right)$.

Independent of the hand representation, participants had a better control over their limb when pointing at the far target.

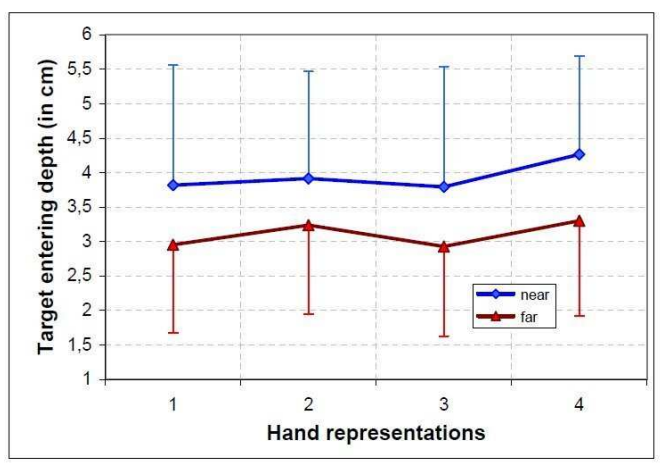

Figure 6: Effect of the hand representation on the target entering depth (means, SDs).

\subsection{Hand movement duration}

Considering action economics, the hand movement duration describes the time a subject has spent between the following two key events:

1. Hand entering the field of view (i.e., hand tracking position intersected with at least one of the two virtual viewing frustums).

2. Stabilising the visual hand on the target square (i.e., last target contact of the displayed hand, incl. hand shifts, before trial end).
An ANOVA indicated that the hand movement duration was not influenced by the hand feedback $(\mathrm{F}(3,33)=1.25 ; \mathrm{p}>0.3)$. But there was a target location effect $(\mathrm{F}(1,11)=10.63$; $\mathrm{p}<0.008)$. That is, pointing towards the close target was significantly faster performed.

\subsection{Hand trajectory length}

The events delimiting the hand trajectory length and hence the second economics measure were the same as for the hand movement duration (see above). No effect on the hand trajectory length was found, neither caused by the hand representation $(\mathrm{F}(3,33)=0.42 ; \mathrm{p}>0.7)$ nor the target location $(\mathrm{F}(1,11)=3.5$; $\mathrm{p}>0.09)$. The latter factor shows only a slight tendency.

\subsection{Questionnaire results}

The analysis of the subjective hand representation evaluation scores was concentrated on visualisation quality, final pointing accuracy, hand movement naturalness and overall comfort.

Subjects had to rank each of these aspects as a function of the hand representation from 1 to 5 (i.e., best to worst, resp.). One participant did not complete the entire evaluation because of strong uncertainties in some cases. His data was excluded from the global analysis and all subquestions concerned.

Table 1: Overall assessment means for the hand representations used (i.e., 1: Best to 5 : Worst).

\begin{tabular}{|c|c|c|c|}
\hline $\begin{array}{c}\text { Real hand } \\
\text { video }\end{array}$ & $\begin{array}{c}\text { Detailed 3D } \\
\text { hand model }\end{array}$ & $\begin{array}{c}\text { Simplified 3D } \\
\text { hand model }\end{array}$ & $\begin{array}{c}\text { Ordinary 3D } \\
\text { pointer arrow }\end{array}$ \\
\hline 1.52 & 1.67 & 2.85 & 3.73 \\
\hline
\end{tabular}

In total, the hand feedback affected the subjective responses significantly $(\mathrm{F}(3,30)=42.01 ; \mathrm{p}<0.0001)$. The Pearson's product moment correlation test showed also a highly significant positive correlation $\left(\mathrm{r}^{2}=0.45 ; \mathrm{t}=12.19 ; \mathrm{p}<0.0001\right)$. This indicates that ranks improved with the realism level of the hand representation (see Table 1). A question effect was not found $(\mathrm{F}(3,30)=0.62, \mathrm{p}>0.6)$.

A post-hoc Newman-Keuls test yielded significantly better total results for the real hand video and the detailed 3D hand model compared to the other two hand representations (i.e., for both: $\mathrm{p}<0.0002$ ). Moreover, the ordinary 3D pointer arrow was rated significantly worse than the simplified $3 \mathrm{D}$ hand model $(\mathrm{p}<0.0003)$. Even if seeing the real hand was generally preferred, no difference was found between the real hand video and the detailed 3D hand model ( $\mathrm{p}>0.6)$.

After having this global acceptance image obtained, we all analysed all subquestions separately. Results are as follows (i.e., main effect and correlation, see also Fig. 7):

1. Visualisation quality: $F(3,33)=24.36 ; p<0.0001$ and $\mathrm{r}^{2}=0.56 ; \mathrm{t}=7.69 ; \mathrm{p}<0.0001$.

2. Final pointing accuracy: $\mathrm{F}(3,33)=5.54 ; \mathrm{p}<0.004$ and $\mathrm{r}^{2}=0.23 ; \mathrm{t}=3.72 ; \mathrm{p}<0.0006$.

3. Hand movement naturalness: $\mathrm{F}(3,30)=30.56$ $\mathrm{p}<0.0001$ and $\mathrm{r}^{2}=0.54 ; \mathrm{t}=7.07 ; \mathrm{p}<0001$.

4. Overall comfort: $\mathrm{F}(3,30)=33.19 ; \mathrm{p}<0.0001$ and $\mathrm{r}^{2}=0.5 ; \mathrm{t}=6.61 ; \mathrm{p}<0.0001$.

Pairwise comparisons (i.e., Fisher's LSD) within each subquestion revealed similar constellations as they were found for the global view (i.e., in most cases: $p<0.001$ or smaller). The only exception was the final pointing accuracy. Here, the simplified 3D hand model was, from a statistical point of view, not evaluated differently from all others. 


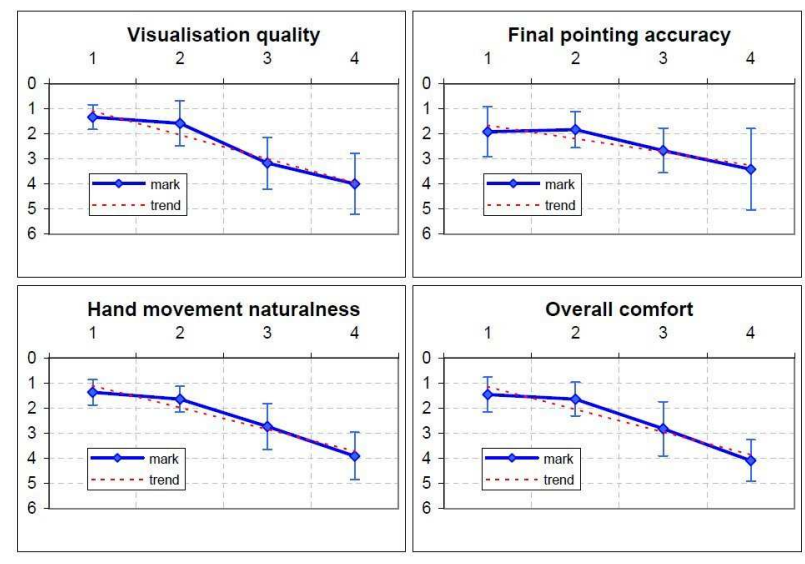

Figure 7: Ranking of the hand representation as a function of the task aspects (means, SDs), with "marks" ranging from 1: Best to 5 : Worst.

\section{Discussion}

The goal of the experiment was to test both the behavioural consequences and user preference hypotheses stated in Section 2.

Hypothesis 1 addressed objective behavioural effect of using different levels of hand representations in a virtual object touching task. The visual hand feedback was spatially constrained to the object's surface. Overall, it seems that the visual limb fidelity has only little influence on motor behaviour under the given conditions. At least, we were unable to show differences for all the variables we have observed. The $3 \mathrm{D}$ pointing stability within a 3 x $3 \mathrm{~cm}$ target was not affected. Lateral target overshooting was found to be the largest for the most abstract hand feedback (i.e., ordinary 3D pointer arrow). This was expected and it confirms previous findings in a way. But, interestingly, several subjects thought to be "more precise" with the arrow because of its sharp end. Global hand movement kinematics did not seem to be affected by the hand representation (see below). So, one possible source for the increased target entering estimation error could have been the non-hand-like shape which prevented a more efficient limb attribution (i.e., a "natural" visuo-motor control). Regarding the Bayesian model of multisensory perception proposed by [19], visual-haptic coupling might have been stronger implying that it was more difficult for the brain to align diverging sensory signals (i.e., here: Visual and proprioceptive hand location). That is, larger hand shifts are more likely which in turn can decrease motor performance referred to co-location. The ordinary $3 \mathrm{D}$ pointer arrow may thus be considered as the least intuitive. A performance benefit of the real hand video compared to the other $3 \mathrm{D}$ hand models of varying visual fidelity could not be shown. There was also no hand representation correlation effect found. We cannot exclude that we did not find effects due to insufficient statistical power. However, it might also be possible that, within the context of the described pointing scenario, an increasingly realistic hand feedback does not improve hand movement precision and stability. A reformulation of the initial hypothesis is hence necessary. The therein mentioned control deficits seem to hold only for very abstract virtual hand substitutes like an arrow.

We argued that the hand representations may neither affect hand movement duration nor hand trajectory length of the visible hand motion towards the targets. It could have been the case that we did not see effects, because first, the main task consisted of touching a visual target with a certain accuracy. Subjects had therefore to focus their attention on the target and peripheral visual guidance of the hand, and so its look, did not play a major role. Or second, the limited fields of view of the VST HMD (i.e., cameras and displays) did not allow viewing the hand earlier during transport. A detailed hand representation might prove beneficial, if the user really focusses on it, for instance, when performing more complex direct virtual object manipulations (e.g., extending [6]). However, if display lags are an issue, one could use a gaze-based level of limb realism to avoid real hand embedding or much more expensive reconstruction techniques as long as the attentional focus is somewhere else.

Concerning target effects, the target entering depth as well as the hand movement duration were statistically dependent on the target location. The coarse hand oscillation and the hand movement trajectory length showed only tendencies. It appears nonetheless to be valid to speak of two widely separated targets that had been introduced to limit adaptive behaviour. The far target revealed better overall stability results. The stereoscopic quality of the hand feedback could have been worse while reaching towards the close target.

In Hypothesis 2, a subjective preference of seeing the own hand over other classical hand representations was expected for several aspects of a near space goal-directed pointing movement (i.e., touching a virtual surface). A lower realism level should lead to a decline in acceptance. Qualitative results show significant preference and correlation effects which largely confirm observations made in previous studies. The more realistic the hand appears, the better users feel when acting in a VE. Although no differences were found between the real hand video and the detailed 3D hand model conditions, participants mostly preferred to see the own hand: "Seems to be very intuitive", "preferred the real hand", "was easy to move towards the red square", "felt to hit the target more quickly with my hand", "can better estimate the hand position, more comfortable", "my hand was the most natural", "because it was my hand", "a better surface understanding and space perception", "comforting to know that I can see my own hand". However, contrary opinions were sometimes expressed as well, for instance: "Preferred the arrow for accuracy reasons", "the arrow for its precision", "the virtual hand, since it 'fits' with the virtual cube", "the 3D hand looked clean (...) did not like the pixelisation of the video hand", "the virtual hand, because it looked '3Dish"'. Beside technical fidelity, it was mostly the precision which was criticised. The accuracy subquestion was actually the only one which showed slightly less distinct hand representation and correlation effects. In summary, the detailed 3D hand model was very often able to compete with the provided real hand video feedback. Reasons for that could have been of technical nature, mainly due to capturing and mixing limitations. Further, the task did not require complex hand or finger movements (e.g., grasping), so that the benefits of a real time hand motion feedback were not fully exploited. In fact, a static gesture was sufficient (but not required!). The initial hypothesis should hence be modified: In the studied case, for a typical pointing-like virtual object touching situation within hand's reach, a high realism level is desirable, but real hand video appears not to be essential. However, seeing the own hand, so far as video, seems nevertheless to convey the subjectively most intuitive form of interaction.

\section{Conclusion AND fUtURe WORK}

We have conducted an experiment to test the hand representation effect on various aspects of a pointing-like near space interaction. Two hypotheses have been stated predicting first, an improved hand movement stability and precision the more realistic the hand appears and second, a better user acceptance. The experimental procedure comprised a goal-directed 3D pointing task and an evaluation questionnaire. Four realism levels of the hand (i.e., from real hand video to an ordinary pointer arrow) were presented while the pointing targets appeared at two spatially varying locations. In the behavioural data analysis, the subjective touching 
event detection was found to be the worst in terms of lateral target overshooting for the most abstract hand representation used in the experiment (i.e., 3D pointer arrow). Limb attribution might have been the weakest here preventing the involvement of "natural" visuo-motor control processes. Among the other more hand-like shaped representations, we could not demonstrate differences in the observed behavioural variables. Questionnaire results show that a higher visual fidelity of the interacting limb is preferred. Subjects further clearly indicated the intuitive character of seeing the own hand. However, an overall ranking did not reveal a statically significant benefit of the real hand video compared to the detailed 3D hand model.

This seems to suggest that, although a high realism level of the limb visualisation improves the subjective feeling of control and comfort as already stated by other researchers, there is no evidence that providing real hand feedback has an impact on motor performance during pointing-like interaction with a quasistatic hand. When designing AR, AV or VR systems, sometimes challenging real limb embedding techniques should thus be counterbalanced with the actual interaction goals - for instance, when object / target selection is the predominant task.

Future work may include the study of the kinematics profiles, adaptation effects and behaviour when being exposed to dynamic visuo-proprioceptive conflicts. We also would like to investigate whether more complex manipulation tasks produce similar results.

\section{Acknowledgements}

This work has partially been supported by the French Research Agency ANR, by the region Rhône-Alpes and by the Intuition NoE EU project. Andreas Pusch has been funded by a grant from the European Community under the Marie-Curie actions within the VISITOR project. The authors would like to thank the subjects for their commitment and patience. The authors are also grateful to the reviewers for their valuable comments.

\section{References}

[1] S.-J. Blakemore and C. D. Frith, "Self-Awareness and Action", In Current Opinion in Neurobiology, 13 (2), pp. 219 - 224, 2003.

[2] H. H. Ehrsson, C. Spence, and R. E. Passingham, "That's My Hand! Activity in Premotor Cortex Reflects Feeling of Ownership of a Limb", In Science Magazine, 305 (5685), pp. 875 - 877, 2004.

[3] D. M. Wolpert, Z. Ghahramani, and M. I. Jordan, "An Internal Model for Sensorimotor Integration", In Science, 269 (5232), pp. $1880-1882,1995$

[4] D. Perani, F. Fazio, N. A. Borghese, M. Tettamanti, S. Ferrari, J. Decety, and M. C. Gilardi, "Different Brain Correlates for Watching Real and Virtual Hand Actions", In Neuroimage, 14 (3), 2001.

[5] J.-L. Vercher, "Perception and Synthesis of Biologically Plausible Motion: From Human Physiology to Virtual Reality.", In Lecture Notes in Computer Science, 3881, pp. 1 - 12, 2006.

[6] B. Lok, S. Naik, M. Whitton, and F. P. Brooks, Jr., "Effects of Handling Real Objects and Avatar Fidelity on Cognitive Task Performance in Virtual Environments", In Proc. of IEEE VR, 2003.

[7] M. Tsakiris and P. Haggard, "The Rubber Hand Illusion Revisited: Visuo-Tactile Integration and Self-Attribution", In J. of Exp. Psych.: Human Perception and Performance, 31, pp. 80 - 91, 2005.

[8] H. J. Snijders, N. P. Holmes, and C. Spence, "Direction-Dependent Integration of Vision and Proprioception in Reaching Under the Influence of the Mirror Illusion”, In Neuropsychologia (ePub), 2006.

[9] P. J. Durlach, J. Fowlkes, and C. J. Metevier, "Effect of Variations in Sensory Feedback on Performance in a Virtual Reaching Task", In Presence: Teleoperators \& Virtual Env., 14 (4), pp. 450 - 462, 2005.

[10] M. Botvinick and J. Cohen, "Rubber Hands "Feel" Touch that Eyes See", In Nature, 391 (756), 1998.

[11] Y. Yuan and Anthony Steed, "Is the Rubber Hand Illusion Induced by Immersive Virtual Reality?", In Proc. of IEEE VR, 2010.

[12] M. R. Mine, F. P. Brooks Jr., and C. H. Sequin, "Moving Objects in Space: Exploiting Proprioception in Virtual-Environment Interaction", In Proc. of SIGGRAPH, 1997.
[13] A. Paljic, S. Coquillart, J.-M. Burkhardt, and P. Richard, "A Study of Distance of Manipulation on the Responsive Workbench(tm)", In Proc. of IPT Symposium 2002, Orlando, USA, 2002.

[14] M. Slater and A. Steed, "A Virtual Presence Counter", In Presence: Teleoperators \& Virtual Environments, 9 (5), pp 413 - 434, 2000.

[15] J. Paillard, "Fast and Slow Feedback Loops for the Visual Correction of Spatial Errors in a Pointing Task: A Reappraisal", In Canadian J. of Physiology and Pharmacology, 74, pp. 401 - 417, 1996.

[16] O. Martin, B. Julian, L. Boissieux, J.-D. Gascuel, and Claude Prablanc, "Evaluating online control of goal-directed arm movement while standing in virtual visual environment", In The Journal of Visualization and Computer Animation, 14, pp. 253 - 260, 2003.

[17] A. Pusch, O. Martin, and S. Coquillart, "HEMP - HandDisplacement-Based Pseudo-Haptics: A Study of a Force Field Application", In Proc. of IEEE 3DUI, 2008.

[18] G. Zachmann and A. Rettig, "Natural and Robust Interaction in Virtual Assembly Simulation", In Eighth ISPE CE: Research and Applications, 2001.

[19] M. O. Ernst, "A Bayesian View on Multimodal Cue Integration", In Human Body Perception From The Inside Out, Ch. 6, pp. 105 - 131, M. Grosjean \& M. Shiffrar (Eds.), 2006. 